\title{
Análise da produção científica de artigos brasileiros sobre EAD
}

\author{
Maria Cristina Rodrigues Azevedo Joly* \\ Maria Aparecida Avelino da Silva** \\ Anelise Silva Dias ${ }^{* * *}$ \\ Ronei Ximenes Martins ${ }^{* * *}$
}

\begin{abstract}
Resumo
Diferentes estudos, orientados por abordagens teóricas distintas, buscam compreender as especificidades da Educação a Distância (EAD) e suas aplicações. Assim, faz-se necessário proceder à revisão de artigos brasileiros acerca do tema buscando conhecer o que se sabe e apontar as lacunas existentes na área. Este estudo visou analisar os artigos científicos brasileiros referentes à EAD no período de 2000 a 2009 presentes nas bases de dados BVS-Psi e Scielo. Considerou-se a palavra-chave Educação a Distância e a presença de resumo para o artigo selecionado. Analisaram-se 306 resumos resultantes da busca inicial e apenas 82 destes referiam-se à área objeto dessa investigação e/ou possuíam resumo para análise. Os artigos analisados eram oriundos de 46 revistas científicas, sendo que a revista Educação e Pesquisa, com Qualis A1, foi a que apresentou maior número de publicações $(N=7)$. A maioria dos estudos realizados foi de autoria múltipla $(70,7 \%)$ e feminina $(62 \%)$, sendo os anos de 2006 e 2007 de maior número de publicações na área. Quanto ao tipo de artigo, houve equivalência entre a quantidade de produções de artigo teórico e de pesquisa, sendo predominantes os estudos na área da Educação. Concluiu-se que, por ser uma área em constante expansão, faz-se necessário um incentivo maior aos estudos sobre o tema bem como maior rigor das revistas no que se refere à qualidade dos resumos publicados, dada a falta de informações fundamentais e de normatização dos mesmos.

Palavras-chave: Aprendizagem virtual; Produção científica; Metaciência; Cientometria.
\end{abstract}

\section{Scientific production analisys of EAD brazilian articles}

\begin{abstract}
Different studies, guided by different theoretical approaches, seek to understand the specifics of Distance Learning (EAD) and its applications. Thus, it is necessary to revise Brazilian articles on the subject seeking to know what we know and point out the gaps in the area. This study analyzes Brazilian scientific articles related to EAD in the period from 2000 to 2009 in the BVS-Psi and Scielo. databases It was considered the keyword "Distance Education" and presence of an abstract for the selected article. We analyzed 306 abstracts resulting from the initial search and only 82 of these referred to the object of this research area and / or possessed abstracts for analysis. The analyzed articles were from 46 scientific journals, being the journal Education and Research, with Qualis A1, the one who showed the highest number of publications $(N=7)$. Most studies were from multiple authors (70.7\%) and female authors (62\%), being the years 2006 and 2007 with the largest number of publications in the area. Regarding the type of article, there was equivalence between the amount of productions of theoretical paper and of research studies, with the ones in the Education area being predominant. It was concluded that, as an area in constant expansion, it is necessary a greater incentive to studies on the subject as well as tightening of magazines with regard to quality of the published abstracts, given the lack of fundamental information and standardization thereof.

Keywords: Virtual Learning, Scientific Production; Metascience; Scientometrics.
\end{abstract}

\section{Introdução}

A ciência engloba um amplo conjunto de atividades realizadas por inúmeras pessoas e instituições, produzindo produtos distintos que repercutem na sociedade de maneira diversificada. Esta tem sido desenvolvida, ao longo da história, propiciando ao conhecimento uma metodologia mais organizada e sistematizada, sendo o século XX, o período de grande crescimento da produção científica (WITTER, 1999).

$\mathrm{O}$ avanço da produção científica promoveu mudanças sociais, políticas, econômicas e pessoais que repercutiram na vida humana, passando a

* Endereço eletrônico: mcrisjoly@ gmail.com

** Endereço eletrônico: maria_ap.silva@ hotmail.com

*** Endereço eletrônico: anelise_dias@yahoo.com.br

**** Endereço eletrônico: rxmartins@ ded.ufla.br 
influenciar suas atividades, tanto em nível social como privado. A produção científica constitui-se em um processo contínuo e dinâmico de descoberta representando um subsídio primordial para o desenvolvimento da ciência (PACHECO, 2005; WITTER, 1996; WITTER, 2002).

Esta refere-se à geração de conhecimento pelo pesquisador e divulgação de seus resultados seja por meio de textos ou produtos tecnológicos para a sociedade. Pela divulgação de seu trabalho, além de contribuir com o avanço de conhecimentos em uma área e tornar acessíveis as informações produzidas, o pesquisador promove o pensamento crítico frente ao que é descoberto, proporcionando novos empenhos e continuidade do processo investigativo (FERREIRA, 2006; PACHECO, 2005).

Considerando a crescente aceleração da produção somada ao alto investimento das mesmas e o impacto causado pela ciência na vida e nas relações humanas, faz-se necessário a avaliação metacientífica sobre o que está sendo produzido nas diversas áreas. $\mathrm{O}$ investimento em trabalhos a partir desta perspectiva pode resultar em informações relevantes que atuam como norteadoras para a ampliação dos trabalhos de pesquisa, reduzindo a ocorrência de estudos simultâneos sobre um mesmo tema, fornecendo e colaborando com reflexões sobre outros elementos importantes para a geração de conhecimento. Assim, o que está sendo publicado, quem publica e que tipo de veículo difunde essa publicação são aspectos importantes para o estudo de produções científicas, já que propiciam uma visão mais ampla a respeito do que tem sido desenvolvido e pesquisado sobre determinado tema (POBLÁCION, WITTER \& SILVA, 2006; WITTER, 2002).

Segundo Silva e Bianchi (2001), as áreas do conhecimento mais evoluídas são as que mais produziram estudos voltados para revisão da sua produção científica, pois estes são utilizados como referência para definir as pesquisas a serem realizadas. Isto confirma a afirmação de Witter (1999), sobre a pesquisa de revisão que sempre pode vir a fornecer alguma informação para a renovação do conhecimento científico e subsidiar pesquisadores e gestores da ciência na busca de recursos necessários para o procedimento científico.

Nesta perspectiva, Ferreira (2006) menciona que a pesquisa de revisão de literatura atua como informante sobre as condições em que a área pesquisada se encontra, identificando as relações, contradições e inconsistências, promovendo sugestões para a resolução de lacunas. No que se refere a análise de produções científicas, Witter (1999) aborda que é pela análise metacientífica que se pode aprimorar o conhecimento disponível, perceber as tendências e as necessidades de uma determinada área, conhecer o nível do conhecimento alcançado, o tipo de informação para os quais os pesquisadores estão dando atenção, as tendências teóricas e metodológicas, conhecer as tecnologias de ensino pesquisadas e os sujeitos enfocados, entre outros aspectos que o pesquisador possa considerar.

Nesse contexto, diversos estudos de análise de produções científicas têm sido realizados nas diversas áreas do conhecimento ao longo dos anos. $\mathrm{Na}$ área da Psicologia, Santos, Oliveira, Joly e Suehiro (2003) analisaram a produção científica sobre psicologia educacional em 304 painéis apresentados no I Congresso Brasileiro de Psicologia, Ciência e Profissão. Verificaram que diferentes temáticas foram focalizadas nos painéis, tendo predominância os trabalhos de relatos de pesquisa, de autoria múltipla e do gênero feminino. Ainda constataram que os resumos apresentaram descrições claras dos procedimentos realizados em que apontavam todos os aspectos necessários ao discurso científico.

No estudo de Joly, Martins, Abreu, Souza e Cozza (2004), os autores localizaram 2239 produções nacionais e internacionais referentes à avaliação psicológica informatizada nos anos de 2000 a 2004. Destes, foram analisados 559 que referiam ao objeto de investigação abordado. Os resultados indicaram equivalência entre a quantidade de publicações nacionais e internacionais sobre o tema, em que a maioria dos estudos foram trabalhos teóricos com discussão voltada sobre a utilização de instrumentos informatizados na avaliação. Houve predominância de autoria múltipla (média de três autores por artigo) e masculina.

Por sua vez, Bruno-Faria, Veiga e Macêdo (2008) realizaram uma revisão de literatura nacional referente à criatividade no contexto das organizações. Estas se encontravam publicadas em revistas de Administração e Psicologia, livros e trabalhos apresentados em um congresso brasileiro da área de Administração entre os anos de 1997 a setembro de 2006. Os autores constataram um aumento das produções sobre o tema nos últimos 10 anos, sendo predominantes estudos descritivos e exploratórios na área de Administração. Concluem, apontando que conceitos como processo criativo, 
criatividade em grupo, entre outros, precisam ser investigados. Apresentam ainda uma agenda de pesquisa que pode auxiliar investigações e o desenvolvimento do tema e, por conseqüência, colaborarão para o aperfeiçoamento da prática no contexto organizacional.

Ainda, Ledesma, Peltzer e Poó (2008) realizaram uma revisão de literatura na base de dados PsycINFO sobre o tema psicologia do trânsito no período de 2000 a 2006. Os autores analisaram a evolução das produções considerando o período revisado, as publicações mais significativas e os países mais produtivos. Também foram analisadas as palavras-chave buscando identificar as principais temáticas e áreas de investigação abordadas pelos estudos. Somou-se a essas análises, a revisão de resumos e artigos completos da revista Transportation Research Part F: Traffic Psychology \& Behaviour a qual pode ser considerada como principal instrumento de publicação da referida temática. Os autores pretenderam contribuir com o desenvolvimento e a publicação de estudos na área e a investigação sobre a mesma a nível latino-americano.

Especificando as pesquisas de análise da produção científica em Educação a Distância, pode ser mencionado o estudo de Camacho (2009) que analisou publicações nacionais que abordavam a Educação a Distância na área da Enfermagem nas bases de dados Lilacs, Scielo e Bdenf (Biblioteca Virtual da Saúde) no período de 2005 a 2009. Foram analisadas 20 referências que abordavam, entre elas, o desenvolvimento de cursos de capacitação e disciplinas de graduação a distância na respectiva área. A autora pôde concluir que há uma evolução da EAD na Enfermagem considerando que as possibilidades de ensino em ambientes on-line são inúmeras e que algumas condições, como a disponibilidade de cursos de capacitação e disciplinas de graduação, somada a tutores que incentivam a interatividade como processo contínuo de aprendizagem são relevantes para o respectivo recurso de formação.

Ainda no que se refere aos trabalhos de análise da produção científica em EAD, pode-se observar que necessitam de mais investimentos para o desenvolvimento e crescimento de produções na área, uma vez que, foi encontrado um único trabalho de origem nacional que aborde a mensuração do referido tema. Nessa direção e ponderando sobre os objetivos da presente pesquisa referente à análise de produções científicas sobre Educação a Distância, é necessário fazer referência e considerações sobre o tema.

A Educação a Distância é definida por pesquisadores (BELLONI, 2001; KEEGAN, 1996; PETERS, 2001; entre outros) e pela legislação educacional (MEC, 2007) como modalidade que se caracteriza pela separação de professores e alunos. Tal separação, de ordem geográfica e ou temporal, requer mediação por tecnologias de informação e comunicação (TIC), utilizadas para entrega de conteúdo e para manutenção do diálogo professoraluno e aluno-aluno (CASTRO \& FERREIRA, 2006; MOORE \& KEARSLEY, 1996; PEDROSO, 2006). Como conseqüência, ocorre alteração na forma com que eles se relacionam uns com os outros e com o conteúdo (ANDERSON \& ELLOUMI, 2004; OLIVEIRA, 1999; PALLOF \& PRATT, 2002; SALMON, 2002).

Avanços recentes na tecnologia educacional permitiram oferta de cursos utilizando a Internet (ANDERSON \& ELLOUMI, 2004; MOORE \& KEARSLEY, 1996; PALLOF \& PRATT, 2002; TAYLOR, 2001). As peculiaridades da modalidade EAD geram diferenças também no projeto e na execução dos cursos (BERNARD, LOU \& ABRAMI, 2002; MOORE, 1993; MOORE \& KEARSLEY, 1996; entre outros). Apesar de todas estas diferenças, é desejável que o desempenho do aluno não seja afetado pela forma com que o curso é oferecido, o que enseja investigação.

No Brasil, até o ano de 1996 não havia dispositivo legal que permitisse oferta de cursos regulares a distância na educação básica ou superior e a existência deles era inexpressiva (MEC/INEP, 2010). O estabelecimento das bases legais para a Educação a Distância se deu a partir do artigo 80 da Lei n. ${ }^{\circ}$ 9.394, de 20 de dezembro de 1996 (BRASIL, 1996), que foi regulamentado inicialmente pelo decreto $\mathrm{n}^{\circ} 2.494$, de 10 de fevereiro de 1998 e por uma sucessão de outros dispositivos (e.g. Decreto 5.622 de 19 de dezembro de 2005). Como efeito do estabelecimento desse marco regulatório, a oferta de cursos se iniciou efetivamente à partir de 2000 , com $0,2 \%$ do total de matrículas em cursos regulares oferecidos no País, evoluindo até $14,1 \%$ em 2009 , o que representa crescimento percentual de $6.950 \%$ desta modalidade em uma década (MEC/INEP, 2010). Em especial, se verifica o acentuado crescimento de $148 \%$ somente em um ano, entre 2005 e 2006.

A expansão da modalidade EAD no Brasil e no âmbito internacional tem motivado diferentes estudos, orientados por abordagens teóricas distintas, que buscam compreender as relações entre 
as especificidades da EAD e suas aplicações e resultados. Como tem característica multidisciplinar, a Educação a Distância é tema de pesquisas em áreas bastante distintas, tais como, a tecnológica (desenvolvimento de tecnologia e inovação; sistemas e ferramentas); de gestão; de políticas públicas, de aspectos educacionais de tecnologia e de ensino-aprendizagem (ZAWACKIRICHTER, 2009).

Considerando essa multiplicidade de temáticas, o interesse da presente pesquisa é principalmente, mas não exclusivamente, a produção relacionada mais aos aspectos educacionais, de tecnologia educacional e de ensino-aprendizagem. Nesse sentido, faz-se necessário proceder a uma revisão da literatura nacional acerca do tema. Este levantamento objetiva dar uma maior visibilidade e destaque às publicações científicas por meio da análise de seu conteúdo e dos periódicos em que se inserem (PACKER \& MENEGHINI, 2006). Ao lado disso, os indicadores encontrados revelam a amplitude da ciência produzida e permitem que esta seja avaliada no tocante ao seu impacto social, cultural e tecnológico (FUJINO, 2006).

Isto posto e dada a necessidade de análise das produções científicas nacionais em Educação a Distância, com ênfase para aspectos educacionais, o presente estudo visou fazer uma revisão na literatura acerca desta temática em bases de dados brasileiras e informatizadas que sistematizam produções nas áreas de psicologia e educação, na década compreendida entre 2000 e 2009. A determinação das bases de dados considera a ênfase aos aspectos educacionais e a determinação temporal se dá em consonância com a estruturação do marco regulatório da modalidade EAD e sua expansão no Brasil, na primeira década deste milénio.

\section{Método}

O presente estudo analisou, a partir das bases de dados BVS-Psi e Scielo, as produções científicas nacionais referentes à Educação a Distância (EAD) no período de 2000 a 2009. Considerou-se a palavra-chave Educação a Distância e a presença de resumo para o artigo selecionado.

\section{Composição da Amostra e Material}

A busca pelas produções científicas foi realizada em janeiro e fevereiro de 2010, nas bases de dados Biblioteca Virtual em Saúde (BVS-Psi) e Scielo por serem as referencias nacionais em Open
Journal Sistem e abarcarem as produções relativas à tecnologia da informação e comunicação, educação e psicologia educacional. Foram localizadas 306 produções científicas referentes a EAD. A partir da análise de conteúdo desses resumos foram retirados 56 que não se referiam a temática em questão (18,3\% do total), 50 resumos de artigos anteriores a 2000 (16,34\% do total), 36 artigos sem resumo (11,8\% do total), 31 artigos repetidos $(10,1 \%$ do total), 22 teses (7,2\% do total), 15 artigos com o mesmo resumo (4,9\% do total), oito artigos com resumos com informações insuficientes $(2,6 \%$ do total), dois relatos de experiência $(0,7 \%$ do total), dois em que a temática do resumo não se referia a palavra-chave do mesmo ( $0,7 \%$ do total $)$, e duas resenhas $(0,7 \%$ do total). Assim, somente 82 artigos (26,8\% do total), referiam-se à área objeto de investigação desta pesquisa e/ou possuíam resumo para análise. Dos resumos analisados e que compuseram a amostra do presente estudo, 9,8\% estavam indexados diretamente no IndexPsi Periódicos Técnico Científicos, $30,5 \%$ no Lilacs, 2,4\% no Pepsic e 57,3\% no Scielo.

\section{Procedimento}

A partir do resultado da busca nas bases de dados, foi feita uma leitura rigorosa de cada um dos resumos para determinar quais pertenciam ao objetivo desse estudo. Os dados foram catalogados em uma planilha composta pelas categorias de análise a serem consideradas para a seleção dos resumos. As categorias selecionadas basearam-se em alguns critérios da metaciência definidos por Witter (1999) como a temática, autoria, discurso e a metodologia. Posteriormente, foi realizada uma análise por três juízes visando a confiabilidade de resultados. Os juízes avaliaram os resumos individualmente e depois conferiram os resultados das análises entre si, com índice de concordância entre eles de $80,0 \%$ por categoria.

No tocante aos critérios definidos para análise pela metaciência, a categoria temática considerou a quantidade de resumos sobre EAD, a área de investigação e o tema principal abordado pelo artigo publicado. Estes critérios foram analisados por meio do título, do conceito exposto no resumo e pelas palavras-chave. Para a análise do tipo da autoria observou-se se o resumo era de autoria múltipla ou individual e, respectivamente, o gênero dos autores. Para o discurso, identificou-se o tipo de artigo (artigo teórico, de pesquisa, revisão de literatura ou estudo de caso) considerando-se para os artigos de pesquisa a presença ou ausência de objetivos, resultados e discussão; para os artigos 
teóricos a presença ou ausência de apresentação da temática, desenvolvimento e conclusão. No quesito metodologia avaliou-se a presença ou ausência de participantes, materiais, procedimentos, o tipo de pesquisa (levantamento, experimental, quaseexperimental ou construção de instrumento) e a análise de dados (quantitativa, qualitativa ou mista). Além dessas categorias, foi realizada uma análise do perfil da revista, a área de conhecimento a que se destina, a classificação Qualis.

\section{Resultados}

Os resultados foram analisados por meio da estatística descritiva buscando apresentar a caracterização das variáveis, perfil das revistas e os critérios da metaciência (temática, autoria, discurso, metodologia e análise de dados). Utilizou-se a Prova Qui-quadrado para verificar possíveis diferenças entre os resultados observados e os esperados no tocante à produção científica para as variáveis consideradas individualmente e associadas ao período de análise das revistas (2000-2010) no presente estudo.

Caracterização geral dos artigos $\underline{\text { analisados }}$

Os 82 artigos analisados encontravam-se distribuídos em 46 revistas científicas, classificadas no Qualis-Periódicos CAPES. Os dados apresentados na Tabela 1 mostram que a maioria $(N=24)$ das produções foram publicadas em revistas de classificação B3, 29,3\% do total das publicações analisadas; a categoria $\mathrm{C}$ contou com menor número de publicações (2,4\%). A revista Educação e Pesquisa, com Qualis A1 da área de Educação, foi a que apresentou maior número de artigos $(N=7)$.

Tabela 1

Distribuição de frequência e porcentagem das revistas em que foram publicados os resumos analisados em função da classificação Qualis

\begin{tabular}{|c|c|c|}
\hline \multirow{2}{*}{$\begin{array}{c}\text { Classificação } \\
\text { Qualis }\end{array}$} & \multicolumn{2}{|c|}{ Número de resumos } \\
\hline & Frequência & Porcentagem \\
\hline A1 & 14 & 17,1 \\
\hline A2 & 15 & 18,3 \\
\hline B1 & 13 & 15,9 \\
\hline B2 & 6 & 7,3 \\
\hline B3 & 24 & 29,3 \\
\hline B4 & 4 & 4,9 \\
\hline B5 & 4 & 4,9 \\
\hline $\mathrm{C}$ & 2 & 2,4 \\
\hline Total & 82 & 100,0 \\
\hline
\end{tabular}

Quanto aos indexadores, a base Scielo foi a que apresentou o maior número de resumos $(57,3 \%)$, seguida pela Lilacs $(30,5 \%)$ e pelo IndexPsi Periódicos Técnico Científicos $(9,8 \%)$. A base Pepsic foi a que apresentou menor quantidade de publicações $(2,4 \%)$. Vale esclarecer que a Lilacs, considerando a etapa de levantamento das produções, foi a que teve o maior número de publicações na área, com um total de 360 artigos. Entretanto, a maioria não apresentava resumo e/ou não se referia ao objeto de estudo desta investigação.

Considerando a quantidades de publicações no período de 2000 a 2010, o período de 2006 a 2007 foi o que possui o maior número de publicações na área $(40,2 \%)$ e o de 2000 a 2001 o menor $(7,3 \%)$. Observa-se ainda, que a frequência das publicações oscilam ao longo dos anos, contradizendo o que é de se esperar para a produção científica de uma área - que com o passar dos anos haja um crescimento sobre o conhecimento adquirido na mesma (Tabela 2 ). 
Tabela 2

Distribuição de frequência e porcentagem dos resumos analisados em função do ano de publicação

\begin{tabular}{lcc} 
Ano de & \multicolumn{2}{c}{ Número de resumos } \\
\cline { 2 - 3 } publicação & Frequência & Porcentagem \\
\hline $2000-2001$ & 6 & 7,3 \\
$2002-2003$ & 16 & 19,5 \\
$2004-2005$ & 7 & 8,5 \\
$2006-2007$ & 33 & 40,2 \\
$2008-2009$ & 20 & 24,4 \\
\hline Total & 82 & 100,0 \\
\hline
\end{tabular}

Focalizando o primeiro critério de análise da metaciência, a temática, procedeu-se a organização dos resumos por área específica e por grande área de conhecimento, classificando-os, portanto em três grupos, a saber, Ciência da Saúde (CS), Ciências Sociais \& Humanidades (S\&H) e Ciências Exatas \& Tecnológicas (E\&T). Os resultados demonstraram equivalência entre a quantidade das produções científicas publicadas na área das Ciências Sociais \& Humanidades e na área da Ciência da Saúde $(42,7 \%)$, em que ambas apresentaram 35 resumos publicados nos últimos dez anos (2000-2009). Na Tabela 3, pode-se observar a freqüência e a porcentagem considerando a área de investigação específica de cada resumo.

Tabela 3

Distribuição de frequência e porcentagem dos resumos analisados em função da área específica

\begin{tabular}{lcc}
\hline \multirow{2}{*}{ Área } & \multicolumn{2}{c}{ Número de resumos } \\
\cline { 2 - 3 } & Frequência & Porcentagem \\
\hline Educação & 23 & 28,1 \\
Enfermagem & 18 & 22 \\
Psicologia & 8 & 9,8 \\
Saúde & 7 & 8,5 \\
Medicina & 6 & 7,3 \\
Tecnologia & 5 & 6,1 \\
Administração & 3 & 3,7 \\
Engenharia de Produção & 3 & 3,7 \\
Odontologia & 2 & 2,4 \\
Física & 2 & 2,4 \\
Radiologia & 1 & 1,2 \\
Fonoaudiologia & 1 & 1,2 \\
Ciência da Informação & 1 & 1,2 \\
Biblioteconomia & 1 & 1,2 \\
Contabilidade & 1 & 1,2 \\
\hline
\end{tabular}

Os estudos foram realizados, em sua maioria, na área da Educação, fato este que já era esperado considerando o objeto de estudo do presente artigo. Os dados evidenciaram a existência de poucos trabalhos realizados sobre EAD o que demonstra a necessidade de mais investigações sobre a área, atentando-se que o período de levantamento foi de 10 anos, no qual houve uma média de, aproximadamente, 8,2 artigos por ano.

Foi verificado também, qual foi o tema principal abordado pelo pesquisador. Isto porque esse item permite observar para qual informação os pesquisadores estão dando mais atenção, bem como as tendências e as necessidades de uma determinada área, afim de, possibilitar estudos que aprimorem o conhecimento disponível (NORONHA et. al., 2006; PACHECO, 2005; WITTER, 1999).

A Tabela 4 mostra a identificação dos 11 temas catalogados a partir dos conceitos que mais apareceram nas produções analisadas. De forma que, o tema EAD foi o que apresentou o maior número de relato de investigações enquanto tema estrito $(36,6 \%)$ e também quando engloba suas aplicações específicas $(72,0 \%)$. 
Tabela 4

Distribuição de frequência e porcentagem dos resumos analisados em função dos temas principais apresentados

\begin{tabular}{lcc}
\hline \multirow{2}{*}{ Temática } & \multicolumn{2}{c}{ Número de resumos } \\
\cline { 2 - 3 } & Frequência & Porcentagem \\
\hline EAD & 30 & 36,6 \\
EAD - formação & 15 & 18,3 \\
EAD - educação continuada & 8 & 9,8 \\
EAD - avaliação & 3 & 3,7 \\
EAD - atitudes & 2 & 2,4 \\
EAD - políticas públicas & 1 & 1,2 \\
TIC & 11 & 13,4 \\
TIC - formação & 9 & 11,0 \\
TIC - avaliação & 1 & 1,2 \\
Estratégias de aprendizagem & 1 & 1,2 \\
Análise de software & 1 & 1,2 \\
\hline Total & 82 & 100,0 \\
\hline
\end{tabular}

Torna-se relevante explicitar os critérios utilizados para as definições dos temas (Tabela 4) e também, a título de ilustração, referenciar algumas produções analisadas, de acordo com a classificação em cada categoria. Classificaram-se como categoria $E A D$ os artigos que abordaram a educação a distância considerando a apresentação de suas definições e sua aplicação frente a cursos e instituições. Um resumo analisado foi o trabalho de Ribeiro e Lopes (2006) que teve como objetivo desenvolver, aplicar e avaliar um curso de EAD por meio da internet; este foi um dos trabalhos que foram considerados como aplicação da EAD. Já o de Kogut (2001) que apresenta um breve histórico da EAD e discussões referentes a problemas e benefícios da mesma, exemplifica o que foi considerado como definições da modalidade em questão.

A categoria $E A D$ - formação caracteriza as produções em que o autor aborda a Educação a Distância como modalidade de formação educacional. Angotti (2006) elaborou um trabalho teórico em que apresentou e discutiu projetos e iniciativas referentes à formação inicial e continuada em Física nas modalidades presencial e a distância.

Por sua vez, a categoria EAD - educação continuada refere-se a estudos que apontam a EAD como recurso para a qualificação de profissionais já graduados. Assim, Preger (2005) realizou uma revisão de literatura sobre os principais endereços eletrônicos referentes a cursos e especializações para a Educação Médica Continuada a Distância com a intenção de facilitar a busca pelos mesmos.

Outra categoria utilizada foi a EAD avaliação que explicita trabalhos que abordaram tanto a avaliação de cursos de EAD, bem como da avaliação como aspecto relevante ao processo educacional e que também deve ser considerada para o presente método educativo. Atentando-se para a avaliação de cursos de EAD, Segenreich (2006) propôs uma discussão referente ao aumento repentino de cursos superiores oferecidos a distância, apontando questões controversas, entre elas a dificuldade da avaliação das finalidades da EAD e seu impacto na formação superior, e sobre documentos legais aprovados e em fase de aprovação.

A EAD - atitudes aborda estudos que tiveram como enfoque atitudes de alunos e professores em relação a cursos de EAD. Steil e Barcia (2006) analisaram as atitudes de alunos e professores em relação a um curso de Engenharia de Produção na modalidade de ensino a distância considerando à interação proporcionada, o papel do professor e as expectativas dos envolvidos em relação ao curso.

Uma outra categoria é a EAD - políticas públicas que foi utilizada para categorizar um estudo teórico de Gatti (2008). O autor realizou uma análise das políticas públicas considerando como o processo de educação continuada, presencial ou a distância, têm sido implementado no contexto das políticas educacionais no Brasil na última década.

A categoria TIC abordou estudos que apresentaram as tecnologias da informação e comunicação como ferramentas de ensino. $\mathrm{O}$ estudo de Nascimento e Trompieri Filho (2002) avaliou a opinião de alunos sobre o uso do correio eletrônico como meio de comunicação entre alunos e professores e o sucesso instrucional dessa utilização na Universidade Federal do Ceará. 
A TIC - formação aborda estudos que utilizam as tecnologias da informação e comunicação (TIC) como recurso ao processo de formação, apresentando assim, a descrição de ambientes virtuais de aprendizagem. Franco, Cordeiro e Castillo (2003), investigaram a incorporação dos ambientes virtuais de aprendizagem na Universidade Estadual de Campinas buscando compreender as conseqüências desse recurso na formação dos alunos e no trabalho dos professores.

Já a TIC - avaliação foi utilizada como definição para o estudo de Laguardia, Portela e Vasconcellos (2007), que enfocou métodos avaliativos das tecnologias empregadas e dos recursos de ensino utilizado nos ambientes virtuais de aprendizagem. Por sua vez, a categoria Estratégia de aprendizagem é um tema que foi abordado em um estudo de Zerbini e Abbad (2008) que construíram e validaram o instrumento Estratégias de Aprendizagem por meio de participantes de um curso nacional a distância via internet.

Por fim, a categoria Análise de software considerou o trabalho de Wataya (2003) em que analisou, a partir do Ambiente Telemático TelEduc, dois softwares Leitores de Tela destinados a Pessoas com Necessidades Especiais Visuais (PNEV's), teve como foco o quanto esses softwares colaboram com a formação a distância dessas pessoas por meio da utilização do ambiente de aprendizagem TelEduc.

Outro critério a ser considerado para a análise metacientífica é o tipo de autoria. Os resultados demonstraram que a maioria dos estudos realizados foi de autoria múltipla $(70,7 \%)$. Em relação ao gênero houve 121 autores femininos e 74 masculinos, sendo que 32 trabalhos tiveram autoria mista.

Ao considerar as análises do discurso, os resultados referentes a tipo de artigo em função das áreas do conhecimento são apresentados na Tabela 5. Notou-se que o número de artigos teóricos é equivalente aos artigos de pesquisa $(N=38)$. Os artigos teóricos compuseram, em sua maioria, estudos realizados na área das $\mathrm{S} \& \mathrm{H}$, sendo o período de 2006 a 2007 de maiores publicações $(N=10)$. Os artigos de pesquisa, por sua vez, possuíram maior divulgação por trabalhos na área da Ciência da Saúde nos últimos quatro anos, de 2006 a 2009. As demais categorias consideradas, estudo de caso e revisão de literatura, apresentaram o menor número de trabalhos.

\section{Tabela 5}

Distribuição de frequência e porcentagem dos resumos analisados em função dos tipos de artigo em função das áreas e anos de publicação

\begin{tabular}{llcccccc}
\hline \multirow{2}{*}{$\begin{array}{l}\text { Tipo de } \\
\text { Artigo }\end{array}$} & \multirow{2}{*}{ Área } & 2000 & 2002 & 2004 & 2006 & 2008 & \multirow{2}{*}{ Total } \\
\cline { 3 - 7 } & & 2001 & 2003 & 2005 & 2007 & 2009 & \\
\hline \multirow{3}{*}{ Teórico } & Saúde & 1 & 2 & 2 & 5 & 0 & 10 \\
& S\&H & 4 & 8 & 1 & 10 & 5 & 28 \\
& Total Parcial & 5 & 10 & 3 & 15 & 5 & 38 \\
\hline \multirow{3}{*}{ Pesquisa } & Saúde & 1 & 3 & 3 & 10 & 10 & 27 \\
& S\&H & 0 & 2 & 1 & 5 & 3 & 11 \\
& Total Parcial & 1 & 5 & 4 & 15 & 13 & 38 \\
\hline \multirow{2}{*}{ Estudo de } & S\&H & & 1 & & 2 & 0 & 3 \\
caso & E\&T & & 0 & & 0 & 1 & 1 \\
& Total Parcial & & 1 & & 2 & 1 & 4 \\
\hline Revisão de & Saúde & & & & 1 & 1 & 2 \\
literatura & Total Parcial & & & & 1 & 1 & 2 \\
\hline
\end{tabular}

Para a análise do discurso foram considerados os resumos de estudo de caso e revisão de literatura como pertencentes a artigos de pesquisa. Foi verificado que todos continham a apresentação do objetivo da pesquisa, $43,2 \%$, não constavam os resultados alcançados e, 61,4\%, não apresentaram a discussão. Por sua vez, quanto à análise da metodologia dos resumos $54,5 \%$ do total de 44 resumos de artigos de pesquisa, não mencionaram os participantes, materiais $\mathrm{e}$ procedimento dos estudos. Vale mencionar que $84,1 \%$ correspondem a artigo de levantamento e $43,2 \%$ refere-se a pesquisas quantitativas. Ao focar os artigos teóricos, as análises evidenciaram que 
todos os resumos contextualizaram a temática investigada. Entretanto, 40,2\% e $30,5 \%$ dos resumos, não expunham o desenvolvimento e a conclusão do estudo, respectivamente.

Visando verificar se houve diferenças nas frequiências observadas em relação às esperadas foi realizado o Teste Qui-quadrado de um critério para cada categoria investigada. Constatou-se que há diferenças estatisticamente significativas para os elementos que compuseram os dados gerais das produções, artigo teórico e perfil da revista.
Destacaram-se também com diferenças significativas o tipo de autoria, tipo de pesquisa e análise de dados. Ao lado disso, não houve diferenças significativas para o gênero de autoria, presença de resultados, discussão, participante, materiais e procedimento (Tabela 6). Além da caracterização geral dos dados alcançados, buscouse realizar uma análise considerando as variáveis por artigo de pesquisa e teórico em função do ano de publicação.

Tabela 6

Estatísticas do Qui-quadrado da caracterização geral dos artigos analisados

\begin{tabular}{|c|c|c|c|c|}
\hline \multicolumn{2}{|c|}{ Caracterização geral } & $\begin{array}{c}\text { Valor do Qui- } \\
\text { quadrado }\end{array}$ & $\begin{array}{l}\text { Graus de } \\
\text { liberdade }\end{array}$ & $\begin{array}{c}\text { Nível de } \\
\text { significância }\end{array}$ \\
\hline \multirow{4}{*}{$\begin{array}{l}\text { Dados gerais } \\
\text { das } \\
\text { produções }\end{array}$} & Área de investigação & 12,902 & 2 & 0,002 \\
\hline & Temática & 106,878 & 10 & 0,000 \\
\hline & Tipo de artigo & 59,854 & 3 & 0,000 \\
\hline & Ano de publicação & 29,585 & 4 & 0,000 \\
\hline \multirow{2}{*}{ Autoria } & Tipo & 14,098 & 1 & 0,000 \\
\hline & Gênero & 3,829 & 2 & 0,147 \\
\hline \multirow{7}{*}{$\begin{array}{l}\text { Artigo de } \\
\text { Pesquisa }\end{array}$} & Presença de resultados & 0,818 & 1 & 0,366 \\
\hline & Presença de discussão & 2,273 & 1 & 0,132 \\
\hline & Participantes & 0,364 & 1 & 0,546 \\
\hline & Materiais & 0,364 & 1 & 0,546 \\
\hline & Procedimento & 0,364 & 1 & 0,546 \\
\hline & Tipo de pesquisa & 82,364 & 3 & 0,000 \\
\hline & Análise de dados & 13,636 & 3 & 0,003 \\
\hline \multirow{2}{*}{$\begin{array}{l}\text { Artigo } \\
\text { Teórico }\end{array}$} & Desenvolvimento & 20,632 & 1 & 0,000 \\
\hline & Conclusão & 3,789 & 1 & 0,052 \\
\hline \multirow{3}{*}{$\begin{array}{l}\text { Perfil da } \\
\text { Revista }\end{array}$} & Área & 38,220 & 2 & $\overline{0,000}$ \\
\hline & Qualis & 38,780 & 7 & 0,000 \\
\hline & Base de dados & 59,561 & 3 & 0,000 \\
\hline
\end{tabular}

\section{Caracterização por tipo de artigo}

\section{Artigos de pesquisa}

Nas análises dos resumos de artigos de pesquisa pode-se observar na Tabela 7 que o período entre 2006 a 2007 foi o que apresentou o maior número de publicações e o período de 2000 a 2001 o menor número. Em relação a classificação Qualis, a categoria B3 foi a que se sobressaiu, sendo as revistas correspondentes predominantemente da área da Saúde. Na classificação A2 também houve uma quantidade considerável de publicações no período analisado. Observou-se, ainda, que ocorreu entre os artigos de pesquisa uma oscilação das publicações ao longo dos anos, tendência esta também verificada quando se analisou a amostra geral de resumos, como anteriormente destacado (Tabela 2). 
Tabela 7

Distribuição de frequência da classificação dos resumos de pesquisa em função do Qualis pelo ano de publicação

\begin{tabular}{|c|c|c|c|c|c|c|}
\hline \multirow{2}{*}{ Qualis } & \multicolumn{5}{|c|}{ Ano de publicação } & \multirow{2}{*}{ Total } \\
\hline & $2000-2001$ & $2002-2003$ & 2004-2005 & $2006-2007$ & $2008-2009$ & \\
\hline A1 & 0 & 0 & 0 & 1 & 0 & 1 \\
\hline A2 & 0 & 2 & 1 & 5 & 3 & 11 \\
\hline B1 & 0 & 0 & 2 & 5 & 3 & 10 \\
\hline B2 & 0 & 0 & 0 & 0 & 2 & 2 \\
\hline B3 & 1 & 3 & 1 & 5 & 6 & 16 \\
\hline B4 & 0 & 1 & 0 & 1 & 0 & 2 \\
\hline B5 & 0 & 0 & 0 & 1 & 1 & 2 \\
\hline Total & 1 & 6 & 4 & 18 & 15 & 44 \\
\hline
\end{tabular}

Em relação a base de dados, a Scielo foi a que obteve o maior número de publicações $(N=24)$ principalmente nos anos de 2006 a $2007(N=13)$. Vale destacar que a base PePsic apresentou o menor número de publicações $(N=2)$, sendo que os dados levantados na base correspondem a $2,4 \%$ do total de resumos analisados. Isto deve-se ao fato do PePsic ter se configurado como base de referencia e indexação recentemente.

Quanto à área de investigação dos artigos de pesquisa, em sua maioria foi da área da Ciência da Saúde $(N=26)$ e em sua minoria das E\&T $(N=7)$. Os resultados evidenciaram ainda, que no decorrer dos últimos dez anos houve um crescimento gradativo em relação às publicações sobre o tema na área da
Saúde, sendo o ápice das publicações os anos de 2006 e 2007.

Considerando a análise das temáticas abordadas foi predominante a categoria EAD (Tabela 8). A temática EAD - formação teve um crescimento de publicações a partir dos anos de 2004 e 2005 com uma freqüência duplicada de artigos entre os períodos posteriores. Abordando a categoria EAD - avaliação observa-se apenas um estudo sobre o tema, em que o fato do mesmo ter sido realizado entre os anos de 2008 e 2009 pode evidenciar o início da preocupação sobre esse estudo que se faz importante, considerando a extrema relevância de avaliar o que tem sido realizado e oferecido sobre a Educação a Distância.

Tabela 8

Distribuição de frequência da temática dos resumos de pesquisa pelo ano de publicação

\begin{tabular}{lcccccc}
\hline & \multicolumn{5}{c}{ Ano de publicação } & \\
\cline { 2 - 6 } Temática & \multicolumn{7}{c}{$2002-$} & $2004-$ & $2006-$ & $2008-$ & Total \\
& $2000-2001$ & 2003 & 2005 & 2007 & 2009 & \\
\hline EAD & 0 & 1 & 0 & 9 & 8 & 18 \\
EAD - formação & 0 & 0 & 1 & 2 & 4 & 7 \\
EAD - educação continuada & 0 & 1 & 1 & 2 & 0 & 4 \\
EAD - avaliação & 0 & 0 & 0 & 0 & 1 & 1 \\
EAD - atitudes & 0 & 0 & 1 & 1 & 0 & 2 \\
TIC & 0 & 4 & 1 & 1 & 0 & 6 \\
TIC - formação & 1 & 0 & 0 & 2 & 1 & 4 \\
Estratégias de aprendizagem & 0 & 0 & 0 & 0 & 1 & 1 \\
Análise de software & 0 & 0 & 0 & 1 & 0 & 1 \\
Total & 1 & 6 & 4 & 18 & 15 & 44 \\
\hline
\end{tabular}

Na Tabela 9 são apresentados os resultados da análise do tipo de autoria. Estes demonstraram que houve predominância de autoria múltipla, 38 resumos, e mista, 19. Vale mencionar que a concentração de autores está entre os anos de 2006 e 2007. 
Tabela 9

Distribuição de frequência do tipo e gênero de autoria dos resumos de pesquisa analisados pelo ano de publicação

\begin{tabular}{llllllll}
\hline \multirow{2}{*}{ Autoria } & \multicolumn{6}{c}{ Ano de publicação } & \\
\cline { 3 - 7 } & & $2000-2001$ & $2002-2003$ & $2004-2005$ & $2006-2007$ & $2008-$ & Total \\
\hline Tipo & Individual & 0 & 1 & 1 & 2 & 2 & 6 \\
& Múltipla & 1 & 5 & 3 & 16 & 13 & 38 \\
\cline { 2 - 7 } & Total & 1 & 6 & 4 & 18 & 15 & 44 \\
\hline \multirow{2}{*}{ Gênero } & Feminino & 0 & 1 & 1 & 5 & 9 & 16 \\
& Masculino & 1 & 2 & 1 & 3 & 2 & 9 \\
& Misto & 0 & 3 & 2 & 10 & 4 & 19 \\
\cline { 2 - 7 } & Total & 1 & 6 & 4 & 18 & 15 & 44 \\
\hline
\end{tabular}

Os dados obtidos a partir da análise do discurso demonstraram que todos os resumos analisados apresentaram o objetivo da pesquisa e os resultados apareceram descritos na maioria dos resumos $(N=25)$. A discussão foi relatada em apenas 17 resumos, sendo que todas as publicações do período de 2002 a 2003 não apresentaram a discussão.

A partir da análise da metodologia pode-se observar que não houve descrição dos participantes, materiais e procedimento em 24 resumos analisados, sendo que o período de 2008 a 2009 foi o que obteve o maior número de publicações sem a exposição clara da metodologia no resumo. Houve ainda, predomínio de pesquisa de levantamento em que a publicação dessa modalidade cresceu e se manteve com a mesma quantidade nos últimos 4 anos. Os demais tipos de pesquisa foram publicados mais no final da década de 2000, mais especificamente nos anos de 2006 a 2007 (experimental e quase-experimental) e nos anos de 2008 a 2009 (construção de instrumento).

Sobre a análise de dados, identifica-se que a maior parte dos resumos analisados são quantitativos. Observou-se a ocorrência do aumento, a partir do período de 2004 a 2005, de publicações qualitativas. Vale mencionar que o número de publicações que não possuem a identificação da análise de dados foi alto $(22,73 \%)$.

\section{Artigos teóricos}

As análises descritivas também foram realizadas para os resumos de artigos teóricos. Por meio dos resultados apresentados na Tabela 10, observa-se a maioria dos artigos $(N=13)$ publicados em revistas A1. Em contrapartida, apenas duas em revistas $\mathrm{C}$.

Tabela 10

Distribuição de frequência da classificação do Qualis dos resumos de artigos teóricos pelo ano de publicação

\begin{tabular}{|c|c|c|c|c|c|c|}
\hline \multirow{2}{*}{ Qualis } & \multicolumn{5}{|c|}{ Ano de publicação } & \multirow[b]{2}{*}{ Tota } \\
\hline & $2000-2001$ & $2002-2003$ & 2004-2005 & $2006-2007$ & $2008-2009$ & \\
\hline A1 & 0 & 6 & 0 & 2 & 5 & 13 \\
\hline $\mathrm{A} 2$ & 0 & 1 & 0 & 3 & 0 & 4 \\
\hline B1 & 0 & 0 & 0 & 3 & 0 & 3 \\
\hline B2 & 1 & 0 & 1 & 2 & 0 & 4 \\
\hline B3 & 0 & 2 & 2 & 4 & 0 & 8 \\
\hline B4 & 1 & 1 & 0 & 0 & 0 & 2 \\
\hline B5 & 1 & 0 & 0 & 1 & 0 & 2 \\
\hline $\mathrm{C}$ & 2 & 0 & 0 & 0 & 0 & 2 \\
\hline Total & 5 & 10 & 3 & 15 & 5 & 38 \\
\hline
\end{tabular}

Os resultados demonstram que a base de dados Scielo foi a que, como identificado para artigos de pesquisa, apresentou o maior número de publicações principalmente no período de 2006 a 2007. No que se refere a área de investigação tanto da revista como dos resumos, as $\mathrm{S} \& \mathrm{H}$ foi a que se 
sobressaiu com 28 e 24 resumos, respectivamente.

Quanto a temática dos resumos analisados, observa-se na Tabela 11 que, a temática predominante é EAD $(N=12)$, seguido por EAD - formação $(N=8)$. Há de se levar em conta, que TIC também é uma temática presente, porém com menor frequência $(N=5)$.

Tabela 11

Distribuição de frequência da temática dos resumos de artigos teóricos pelo ano de publicação

\begin{tabular}{lcccccc}
\hline \multirow{2}{*}{ Temática } & \multicolumn{7}{c}{ Ano de publicação } & & \\
& $2000-$ & $2002-$ & $2004-$ & $2006-$ & $2008-$ & Total \\
\hline EAD & 2001 & 2003 & 2005 & 2007 & 2009 & \\
EAD - formação & 2 & 3 & 2 & 3 & 2 & 12 \\
EAD - educação continuada & 1 & 0 & 1 & 4 & 2 & 8 \\
EAD - avaliação & 0 & 1 & 0 & 3 & 0 & 4 \\
EAD - políticas públicas & 0 & 0 & 0 & 2 & 0 & 2 \\
TIC & 0 & 0 & 0 & 0 & 1 & 1 \\
TIC - formação & 2 & 1 & 0 & 2 & 0 & 5 \\
TIC - avaliação & 0 & 5 & 0 & 0 & 0 & 5 \\
\hline Total & 0 & 0 & 0 & 1 & 0 & 1 \\
\hline
\end{tabular}

Com relação ao tipo de autoria, pode-se verificar por meio dos dados apresentados na Tabela 12 que houve uma predominância da autoria múltipla $(N=20)$. Quanto ao gênero, observa-se que a maioria das investigações foram realizadas por pesquisadores femininos $(N=15)$ e 13 resumos tiveram autoria mista.

Tabela 12

Distribuição de frequência do tipo e gênero de autoria dos resumos de artigos teóricos analisados pelo ano de publicação

\begin{tabular}{llcccccc} 
& & \multicolumn{7}{c}{ Ano de publicação } & \multirow{2}{*}{ Total } \\
\cline { 3 - 7 } Autoria & $2000-$ & $2002-$ & $2004-$ & $2006-$ & $2008-$ & \\
\hline \multirow{2}{*}{ Tipo } & 2001 & 2003 & 2005 & 2007 & 2009 & \\
& Individual & 5 & 4 & 0 & 5 & 4 & 18 \\
& Múltipla & 0 & 6 & 3 & 10 & 1 & 20 \\
\cline { 2 - 7 } & Total & 5 & 10 & 3 & 15 & 5 & 38 \\
\hline \multirow{2}{*}{ Gênero } & Feminino & 2 & 5 & 1 & 6 & 1 & 15 \\
& Masculino & 3 & 0 & 0 & 4 & 3 & 10 \\
& Misto & 0 & 5 & 2 & 5 & 1 & 13 \\
\cline { 2 - 7 } & Total & 5 & 10 & 3 & 15 & 5 & 38 \\
\hline
\end{tabular}

Ao considerar a análise de discurso, todos os resumos teóricos analisados contaram com uma apresentação da temática, mas na maioria deles não houve a presença do desenvolvimento $(N=33)$, nem da conclusão $(N=25)$. Desta forma, de uma maneira geral, não houve a apresentação de todas as informações relevantes ao discurso científico na maioria dos resumos analisados, o que é de fundamental importância em se tratando de artigo teórico, dada ter como base a reflexão e/ou discussão da temática apresentada.

\section{Discussão}

À guisa de síntese, constatou-se na análise da produção científica em EAD, no período de 2000 a 2009, que dos 82 resumos analisados, $57,3 \%$ encontravam-se na base de dados Scielo. 
Distribuídas em 46 revistas, a maioria das produções foram publicadas em revistas de classificação Qualis B3 (29,3\%). É importante destacar que as produções tem visibilidade no cenário nacional por se encontrarem na Scielo, favorecendo assim, investigações que se complementem possibilitando, de fato, um avanço real da área (FERREIRA, 2006; PACHECO, 2005). A despeito disso, é preocupante o fato destas estarem em revistas Qualis B3, em sua maioria, pois de acordo com Packer e Meneghini (2006) e Fujino (2006) o impacto social e científico das informações é dependente deste critério de inserção. Cabe também uma investigação futura, de caráter mais qualitativo, acerca dos motivos que levaram os autores a publicar, com mais freqüência, suas pesquisas em revista B3; era de se esperar, considerando Packer e Meneghini (2006), que as informações dos estudos em análise tivessem maior visibilidade e impacto científico e profissional, em função de quão inéditas elas são.

Em relação ao número de publicações, o período de 2006 a 2007 foi o que apresentou maior quantidade de artigos em EAD (40,2\%), revelando uma descontinuidade do processo de produção científica da área, o que pode vir a comprometer a geração de conhecimento científico para além da área específica em análise no presente estudo, de acordo com a perspectiva de Witter $(1996 ; 2002)$ e Pacheco (2005).

Considerando a área de investigação, houve equivalência entre a quantidade de produções na área das S\&H e na Ciência da Saúde (42,7\%). A temática mais investigada foi a $\operatorname{EAD}(36,6 \%)$, referente a trabalhos que apresentaram a EAD a partir de suas definições e aplicação frente a cursos e instituições, indicando, por um lado, os avanços quanto a aplicação da tecnologia na educação visando o desenvolvimento de cursos pela Internet (ANDERSON \& ELLOUMI, 2004; MOORE \& KEARSLEY, 1996; PALLOF \& PRATT, 2002; TAYLOR, 2001). De outro, a importância de pesquisas voltadas para as características de aprendizagem (BELLONI, 2001; KEEGAN, 1996; PETERS, 2001) e interação em cursos a distância (ANDERSON \& ELLOUMI, 2004; CASTRO \& FERREIRA, 2006; OLIVEIRA, 1999; PALLOF \& PRATT, 2002; PEDROSO, 2006; SALMON, 2002), dada suas especificidades (BERNARD, LOU \& ABRAMI, 2002; MOORE, 1993; MOORE \& KEARSLEY, 1996).

Constatou-se ainda, que a maioria dos estudos realizados foi de autoria múltipla $(70,7 \%)$ e feminina (62\%), o que pode indicar uma predominância de produção científica na área por grupo de pesquisas os quais, por serem de caráter educacional, contam com maioria de pesquisadoraseducadoras. Quanto ao tipo de artigo, houve equivalência entre a quantidade de produções de artigo teórico e de pesquisa $(N=38)$, sendo predominantes os estudos na área da Educação, assinalando uma importante tendência acerca da evolução da área (FERREIRA, 2006; PACHECO, 2005, WITTER, 1999).

A caracterização dos artigos de pesquisa evidenciou que o período de 2006 a 2007 e a base de dados Scielo obteve o maior número de publicações, sendo que a maioria em revistas Qualis B3. Este dado pode indicar que a publicação pelo MEC (2007) da legislação para EAD determinou reformulações por parte das instituições educacionais que ofertaram cursos nesta modalidade, imprimindo, deste modo, maior lentidão à produção científica.

A maioria dos estudos é da área da Ciência da Saúde e a temática mais estudada foi EAD, corroborando os dados obtidos no estudo de Camacho (2009) que concluiu que há uma evolução da EAD na área da enfermagem. Houve predominância de autoria múltipla, 38 resumos, e mista, 19. O discurso evidenciou que todos os resumos apresentaram o objetivo da pesquisa. Considerando os resultados apareceram descritos na maioria das publicações $(N=25)$. A discussão foi descrita em apenas 17 resumos, sendo que as produções do período de 2002 a 2003 não a apresentaram. Por sua vez, a metodologia não apresentou a descrição dos participantes, materiais e procedimentos em 24 resumos, evidenciando que não houve a exposição clara da metodologia utilizada. Tendo por referência a perspectiva da metaciência (WITTER, 1999; 2002) e do conteúdo mínimo necessário para a divulgação científica, fica evidente a escassez de informação da área (POBLÁCION, WITTER \& SILVA, 2006). Quanto ao tipo de pesquisa e a análise de dados, respectivamente, foi predominante a pesquisa de levantamento, 37 resumos, e a análise quantitativa, 19 resumos.

Por sua vez, os artigos teóricos foram publicados em sua maioria em revistas de classificação A1 $(N=13)$. Novamente a Scielo obteve o maior número de publicações, 23 resumos. Isto revela um avanço da discussão teórica para $\mathrm{EAD}$, apesar de não terem sido encontrados estudos de revisão da literatura (SILVA \& BIANCHI, 
2001). A área de investigação dos trabalhos foi predominante a das $\mathrm{S} \& \mathrm{H}(N=24)$. A temática EAD apresentou mais estudos $(N=12)$. Todos os resumos analisados descreveram a apresentação da temática, porém na maioria não houve a presença do desenvolvimento $(N=33)$ e da conclusão $(N=25)$ o que demonstra, que de uma maneira geral nos resumos analisados, não houve a apresentação de todas as informações relevantes ao discurso científico. Tais resultados são semelhantes aos encontrados na análise dos resumos referentes a artigos de pesquisa, cabendo, pois, as mesmas considerações anteriormente já postas.

Os resultados do Teste Qui-quadrado demonstraram diferenças estatisticamente significativas para os elementos área de investigação, temática, tipo de artigo, ano de publicação, artigo teórico, perfil da revista, tipo de autoria, tipo de pesquisa e análise de dados. $\mathrm{O}$ que não foi observado para o gênero de autoria, presença de resultados, discussão, participante, materiais e procedimento.

Em suma, estas informações são importantes a medida que demonstram o que tem sido desenvolvido e pesquisado sobre o tema, bem como revelam que é necessário aprimorar o conhecimento disponível propiciando ao pesquisador referência para definir futuros temas e construtos a serem investigados (FERREIRA, 2006; POBLÁCION, WITTER \& SILVA, 2006; SILVA \& BIANCHI, 2001; WITTER, 1999, 2002).

\section{Considerações Finais}

Tendo em vista os resultados alcançados pode-se concluir que por ser uma área em constante expansão, faz-se necessário um incentivo maior aos estudos sobre o tema. Considerando que a maioria dos estudos refere-se à apresentação da EAD a partir de suas definições e aplicação frente a cursos e instituições, aconselha-se que haja pesquisas referentes a temas que carecem de estudos como a avaliação de cursos de EAD, bem como a avaliação do desempenho dos alunos formados por esse recurso, temas esses de extrema relevância para a verificação da qualidade do mesmo.

Salienta-se ainda, maior rigor por parte das revistas no que se refere à qualidade dos resumos publicados e a necessidade de normatização dos mesmos, dada a falta de informações fundamentais ao discurso científico. Tal fato pode ser um dos aspectos limitadores ao crescimento da área investigada já que dificulta e inviabiliza a análise de sua produção científica.

\section{Referências}

ANDERSON, Terry; ELLOUMI, Fathi. Theory and Practice of Online Learning. Canadá: Athanasca University, 2004. Disponível em: <http:/cde.athabascau.ca/online_book>. Acessado em: 02 março 2010.

ANGOTTI, José André Peres. Desafios para a formação presencial e a distância do físico educador. Revista Brasileira de Ensino de Física, [S.I.], v. 28, n. 2, p. 143-150, 2006.

BELLONI, Maria Luiza. Educação a Distância. 2. ed. Campinas: Autores Associados, 2001.

BERNARD, Robert M.; LOU, Yiping; ABRAMI, Philip. C. Comparing Distance Education and Traditional Instruction: An "In-progress" MetaAnalysis of the Empirical Literature. Review of Educational Research, [S.I], p. 345-359, 2002.

BRASIL, MEC - INEP. Censo da Educação Superior de 2009. Resumo Técnico, 34. Brasília, DF, Brasil: MEC. 2010.

BRASIL.. Lei de Diretrizes e Base da Educação. Lei No 9394 . Brasilia, DF, Brasil: MEC.1996.

BRASIL, Decreto Presidencial n. ${ }^{\circ} 5622$ de 19 de dezembro de 2005. Disponível em: http://www.mec.gov.br. Acesso em 17 dez. 2013.

BRUNO-FARIA, Maria de Fátima; VEIGA, Heila Magali da Silva; MACÊDO, Laura Ferreira. Criatividade nas organizações: análise da produção científica nacional em periódicos e livros de Administração e Psicologia. rPOT, v.8, n. 1, p. 142-163, jan./jun., 2008.

CAMACHO, Alessandra Conceição L. F. Análise das publicações nacionais sobre educação à distância na enfermagem. Revista Brasileira de Enfermagem, v. 62, n. 4, p. 588-593, 2009.

CASTRO, Márcia Nardelli Monteiro de; FERREIRA, Luciana Dias Vieira. TD\&E a distância: múltiplas mídias e clientelas. In: BORGES-ANDRADE, Jairo Eduardo; ABBAD, 
Gardênia da Silva; MOURÃO, Luciana (Orgs.). Treinamento, desenvolvimento e educação em organização e trabalho: fundamentos para a gestão de pessoas. 1. ed. Porto Alegre: Artmed, 2006, p. 323-339.

FERREIRA, Adriana. A. Produção científica sobre idoso na PsycINFO (2003). In: WITTER, Geraldina. P. (Org.). Envelhecimento: referenciais teóricos e pesquisas. 1. ed. Campinas, SP: Editora Alínea, 2006, p. 177-209.

FRANCO, Marcelo A.; CORDEIRO, Luciana M.; CASTILLO, Renata A. F. del. O ambiente virtual de aprendizagem e sua incorporação na Unicamp. Educação e Pesquisa, v. 29, n. 2, p. 341-353, jul./dez., 2003.

FUJINO, Ana. Avaliação dos impactos da produção científica na produção tecnológica. In: POBLÁCION, Dinah A.; WITTER, Geraldina P.; SILVA, José Fernando M. da (Orgs.). Comunicação e produção científica. 1. ed. São Paulo: Angellara, 2006, p. 371-386.

GATTI, Bernadete A. Análise das políticas públicas para formação continuada no Brasil, na última década. Revista Brasileira de Educação (online), v. 13, n. 37, p. 57-70, 2008.

JOLY, Maria Cristina R. A.; MARTINS, Ronei X.; ABREU, Mirthis C.; SOUZA, Paulo Roberto R..; COZZA, Heitor F. P. Análise da Produção Científica em Avaliação Psicológica Informatizada. Avaliação Psicológica, v. 3, n. 2, p. 121-129, 2004.

KEEGAN, Desmond. Foundations of Distance Education. 3. ed. London e New York: Routledge, 1996, 218p.

KOGUT, Eliane Chermann. Educação à distância: presente/futuro ou futuro/presente. Encontro, n. 6, p. 29-31, jan./dez., 2001.

LAGUARDIA, Josué; PORTELA, Margareth Crisóstomo; VASCONCELLOS, Miguel Murat. Avaliação em ambientes virtuais de aprendizagem. Educação a Pesquisa, v. 33, n. 3, p. 513-530, 2007.

LEDESMA, Rubén; PELTZER, Raquel; POÓ, Fernando. Análisis de la producción en Psicología del Tránsito mediante PsycINFO (2000-2006). PSIC - Revista de Psicologia da Vetor Editora, v. 9, n.
1, p. 11-24, jan./jun., 2008.

MEC. Ministério de Educação (2007). Regulamentação da EAD no Brasil. Disponível em: <portal.mec.gov.br/sesu/arquivos/pdf/TREAD. pdf>. Acessado em: 14 novembro 2009.

MOORE, Michel G. Teoria da distância transacional. In: KEEGAN, D. Theoretical Principles of Distance Education. Londres: Routledge, 1993. p. 22-38. Trad. Wilson Azevedo. Disponível em: 〈 http://www.abed.org.br/>. Acessado em: 24 janeiro 2010.

MOORE, Michel G.; KEARSLEY, Greg. Distance Education: a systems view. Belmont (CA): Wadsworth, 1996.

NASCIMENTO, Raimundo Benedito; TROMPIERI FILHO, Nicolino. Correio eletrônico como recurso didático no ensino superior - $\quad 0$ caso da Universidade Federal do Ceará. Ciência da Informação, v. 31, n. 2, p. 86-97, 2002.

NORONHA, Ana Paula Porto, et al. Análise de Teses e Dissertações em Orientação Profissional. Revista Brasileira de Orientação Profissional, v. 7, n. 2, p. 1-10, 2006.

OLIVEIRA, José Palazzo M. Sistemas de Ensino na Internet - Projeto Tapejara. Porto Alegre: UFRGS, $1999 . \quad$ Disponível em: <tecnologiaedu.us.es/bibliovir/pdf/19art1.pdf>.

Acessado em: 18 janeiro 2010.

PACHECO, Elisabeth M. C. Produção científica e avaliação psicológica. In: WITTER, Geraldina. P. (Org.). Metaciência e Psicologia. 1. ed. Campinas, SP: Editora Alínea, 2005. p. 7-33.

PACKER, Abel. L.; MENEGHINI, Rogério. Visibilidade da produção científica. In: POBLÁCION, Dinah A.; WITTER, Geraldina P.; Silva, José Fernando M. da (Orgs.). Comunicação e produção científica. 1. ed. São Paulo: Angellara, 2006. p. 235-260.

PALLOFF, Rena M.; PRATT, Keith. Construindo Comunidades de Aprendizagem no Ciberespaço: estratégias eficientes para sala de aula on-line. Tradução de Vinícius Figueira. Porto Alegre: ARTMED, 2002. 
PEDROSO, Gelta. M. J. Fatores críticos de sucesso na implementação de programas de EAD via internet nas universidades comunitárias. Florianópolis, SC, 2006. 147. Tese de doutorado, Departamento de Engenharia de Produção de Sistemas, Universidade Federal de Santa Catarina.

PETERS, Otto. Didática do ensino a distância: experiências e estágio. São Leopoldo: Unisinos, 2001.

POBLÁCION, Dinah A.; WITTER, Geraldina P.; SILVA, José Fernando M. da. Comunicação \& produção científica: contexto, indicadores e avaliação. São Paulo: Angellara Editora, 2006. p. 428.

PREGER, Claus Michael. Educação médica continuada a distância em endocrinologia e metabologia. Arquivos Brasileiros de Endocrinologia e Metabologia, v. 49, n. 4, p. 584595, 2005.

RIBEIRO, Maria Andréia S.; LOPES, Maria Helena B. M. Desenvolvimento, aplicação e avaliação de um curso à distância sobre tratamento de feridas. Revista Latino-Americana de Enfermagem, v. 14, n. 1, p. 77-84, 2006.

SALMON, Gilly. E-tivities: the key to active online learning. Londres: Kogan Page, 2002.

SANTOS, Acácia A. A.; OLIVEIRA, Katya L.; JOLY, Maria Cristina R. A.; SUEHIRO, Adriana C. B. I Congresso Nacional de "Psicologia- Ciência e Profissão": O que tem sido feita na Psicologia Educacional. Psicologia Escolar e Educacional, v. 7, n. 2, p. 135-144, 2003.

SEGENREICH, Stella Cecilia D. Desafios da educação à distância ao sistema de educação superior: novas reflexões sobre o papel da avaliação. Educar, n. 28, p. 161-177, 2006.
SILVA, José Aparecido; BIANCHI, Maria de Lourdes Pires. Cientometria: A métrica da ciência. Paidéia, v. 11, n. 20, p. 5-10; 2001.

STEIL, Andrea Valéria; BARCIA, Ricardo Miranda. Atitudes de alunos e professores com relação a cursos de mestrado em engenharia de produção a distância. Gestão \& Produção, v. 13, n. 1, p. 141-149, 2006.

TAYLOR, Jim C. Fifth generation of distance education, 2001. Disponível em: <www.dest.gov.au/archive/highered/hes/hes40/hes4 0.pdf.> Acessado em: 10 abril 2010.

ZERBINI, Thaís; ABBAD, Gardênia. Estratégias de aprendizagem em curso a distância: validação de um escala. Psico-USF, v. 13, n. 2, p. 177-187, jul./dez., 2008.

ZAWACKI-RICHTER, Olaf. Research areas in distance education: A Delphi study. International Review of Research in Open and Distance Learning, v. 10, n. 3, 2009.

WATAYA, Roberto Sussumu. O uso de leitores de tela no TelEduc. Interface - Comunicação, Saúde e Educação, v. 10, n. 19, p. 227-242, 2006.

WITTER, Geraldina P. Avaliação da produção científica sobre leitura na Universidade (1989/1994). Psicologia Escolar e Educacional, v. 1, n. 1, p. 31-38, 1996.

WITTER, Geraldina. P. Metaciência e Leitura. In: WITTER, Geraldina P. (Org.). Leitura: Textos e pesquisas. 1. ed. Campinas, SP: Editora Alínea, 1999, p. 13-22.

WITTER, Geraldina. P. Produção científica sobre Estresse e Prevenção. In: WITTER, Geraldina P. (Org.) Psicologia: tópicos gerais. 1. ed. Campinas, SP: Editora Alínea, 2002, p. 213-238.

\section{Sobre os autores:}

Maria Cristina Rodrigues Azevedo Joly: Doutora em Psicologia da Aprendizagem e do Desenvolvimento Humano pela Universidade de São Paulo. Pesquisadora colaboradora sênior do Programa de Pós-Graduação Stricto Sensu Desenvolvimento Humano e Saúde da Universidade de Brasília.

Maria Aparecida Avelino da Silva: Psicóloga pela Universidade São Francisco. 
Anelise Silva Dias: Doutora pelo Programa de Pós-Graduação Stricto Sensu da Universidade São Francisco. Docente da Universidade Paulista.

Ronei Ximenes Martins: Doutor em Psicologia pela Universidade São Francisco. Docente do Departamento de Educação e coordenador de Educação a Distância da Universidade Federal de Lavras.

Recebido em 10/02/2013

Aprovado em 27/09/2013 